\title{
Effect of an anisotropic escape mechanism on elliptic flow in relativistic heavy-ion collisions
}

\author{
Amaresh Jaiswal ${ }^{1}$ and Partha Pratim Bhaduri ${ }^{2}$ \\ ${ }^{1}$ School of Physical Sciences, National Institute of Science Education and Research, HBNI, Jatni 752050, India \\ ${ }^{2}$ Variable Energy Cyclotron Centre, HBNI, 1/AF Bidhan Nagar, Kolkata 700 064, India
}

(Received 8 December 2017; published 16 April 2018)

\begin{abstract}
We study the effect of an anisotropic escape mechanism on elliptic flow in relativistic heavy-ion collisions. We use the Glauber model to generate initial conditions and ignore hydrodynamic expansion in the transverse direction. We employ the Beer-Lambert law to allow for the transmittance of produced hadrons in the medium and calculate the anisotropy generated due to the suppression of particles traversing through the medium. To separate non-flow contribution due to surface bias effects, we ignore hydrodynamic expansion in the transverse direction and consider purely longitudinal boost-invariant expansion. We calculate the transverse momentum dependence of elliptic flow, generated from an anisotropic escape mechanism due to surface bias effects, for various centralities in $\sqrt{s_{N N}}=200 \mathrm{GeV} \mathrm{Au}+\mathrm{Au}$ collisions at the Relativistic Heavy Ion Collider and $\sqrt{s_{N N}}=2.76 \mathrm{TeV} \mathrm{Pb}+\mathrm{Pb}$ collisions at the Large Hadron Collider. We find that the surface bias effects make a sizable contribution to the total elliptic flow observed in heavy-ion collisions, indicating that the viscosity of the QCD matter extracted from hydrodynamic simulations may be underestimated.
\end{abstract}

DOI: 10.1103/PhysRevC.97.044909

\section{INTRODUCTION}

High energy heavy-ion collisions aim to create and study different phases of quantum chromodynamics (QCD) at extremely high temperature and density [1]. Observation of transverse flow, mass ordering, and anisotropic flow in experimental data at the Relativistic Heavy Ion Collider (RHIC) and the Large Hadron Collider (LHC) indicates the presence of the hydrodynamic phase in the evolution. Agreement of the experimental data with hydrodynamics-inspired models suggests that the system is locally thermalized and possesses a collective flow velocity due to the buildup of extremely high pressure, which drives the system to expand at relativistic speed. Indeed relativistic hydrodynamics has been applied quite successfully to model high energy heavy-ion collisions [2-5]. Moreover, the application of relativistic viscous hydrodynamics to heavy-ion collisions has evoked widespread interest ever since the ratio of shear viscosity to entropy density, $\eta / s$, estimated from the analysis of anisotropic flow data was found to be close to the conjectured lower bound of $1 / 4 \pi$ [6]. This led to the claim that the quark-gluon plasma (QGP) formed in relativistic heavy-ion collisions was the most perfect fluid ever observed.

No other theoretical construct has been able to describe the multitude of experimental data on heavy-ion collisions at the same level of accuracy as relativistic hydrodynamics [7-11]. However, some theoretical ideas are able to mimic certain types

Published by the American Physical Society under the terms of the Creative Commons Attribution 4.0 International license. Further distribution of this work must maintain attribution to the author $(s)$ and the published article's title, journal citation, and DOI. Funded by $S C O A P^{3}$. of hydrodynamic signals [12]. For instance, it was proposed that surface bias effects originating from the escape mechanism of the partons may make a large contribution to anisotropic flow even if the system interactions are small [10]. This challenges the current understanding of the anisotropic flow generated from hydrodynamic pressure gradients. Moreover, if anisotropic flow originating from surface bias effects is indeed larger or even comparable to hydrodynamically driven collective flow, then the extracted $\eta / s$ is severely underestimated. Since escape is inevitable for a transient colliding system, it is imperative to examine the possible role of the escape mechanism on anisotropic flow within the framework of relativistic hydrodynamics.

It is generally perceived that large elliptic anisotropy in momentum of the observed particles, also known as elliptic flow, can only be generated in heavy-ion collisions where the size of the medium is large enough to admit a hydrodynamic description. Of particular interest are non-central heavy-ion collisions where the overlap volume of the colliding nuclei is anisotropic in the transverse plane (perpendicular to the beam). The pressure gradient due to the initial geometrical anisotropy would generate anisotropic expansion and finalstate elliptic flow. Indeed large elliptic flow has been measured in heavy-ion collisions at RHIC and LHC, which can be well explained within the framework of relativistic hydrodynamics. However, recent particle correlation data hint at similar elliptic flow in high multiplicity $d+\mathrm{Au}$ collisions at RHIC [13] and $p+p$ and $p+\mathrm{Pb}$ collisions at the LHC [14-24]. While hydrodynamics seems to describe the experimental data well, suggesting that these small-system collisions might create QGP, it is in contrast to general expectations based on the argument that equilibration cannot be achieved in such small systems and therefore hydrodynamics might not be applicable. However, an anisotropic escape mechanism due to surface bias 
effects might play a dominant role in generating large elliptic flow in these small-system collisions [25,26].

The consequences of a freeze-out criterion for heavyion collisions, based on pion escape probabilities from the rapidly expanding hot and dense medium, and the influence of expansion and scattering rate on the escape probability were studied before in Ref. [27]. Moreover, it was also found that a single collision per particle, on average, is already enough to generate sizable elliptic flow, with mass ordering between the species [28]. This was indicative of the role of the escape mechanism in mimicking certain types of hydrodynamic signals. In the context of heavy flavors, the propagation of charm and bottom quarks through an ellipsoidal QGP was studied in Refs. [29,30]. The elliptic flow thus obtained was due to a difference in energy loss in the transverse plane originating from inequal path lengths in an ellipsoidal QGP. To this end, an experimentally accessible observable, which discriminates between collective and non-collective contributions to the observed elliptic flow, was also proposed in Ref. [31].

In this paper, we study the effect of an escape mechanism due to shape anisotropy on elliptic flow in relativistic heavy-ion collisions. We use the Glauber model to generate initial conditions and ignore hydrodynamic expansion in the transverse direction to separate out the non-flow contribution due to surface bias effects. We employ the Beer-Lambert law to allow for the transmittance of produced hadrons in the medium and calculate the anisotropy generated due to the suppression of particles traversing through the medium. While the analysis in Ref. [10] was performed within the framework of transport theory, we consider longitudinal boost-invariant hydrodynamic expansion in order to estimate the surface bias effects. We calculate the transverse momentum dependence of elliptic flow, generated from an anisotropic escape mechanism due to surface bias effects, for various centralities in $\sqrt{s_{N N}}=200$ $\mathrm{GeV} \mathrm{Au}+\mathrm{Au}$ collisions at RHIC and $\sqrt{s_{N N}}=2.76 \mathrm{TeV}$ $\mathrm{Pb}+\mathrm{Pb}$ collisions at LHC. We find that the surface bias effects have a sizable contribution to the total elliptic flow observed in these heavy-ion collisions. This indicates that the viscosity of the QCD matter, extracted from hydrodynamic simulations, may be underestimated.

\section{THE MODEL}

We work in the Milne coordinate system $\left(\tau, x, y, \eta_{s}\right)$, where $\tau=\sqrt{t^{2}-z^{2}}$ and $\eta_{s}=\tanh ^{-1}(z / t)$. The metric tensor for this coordinate system is $g_{\mu \nu}=\operatorname{diag}\left(1,-1,-1,-\tau^{2}\right)$. For longitudinal boost-invariant flow, i.e., $v^{z}=z / t$, the fluid fourvelocity is given by $u^{\mu}=(1,0,0,0)$.

To separate the surface bias effects due to source shape anisotropy on transverse momentum anisotropy of the observed particles, we assume vanishing fluid velocity in the transverse direction. This implies that the transverse energy density distribution of the system retains the initial shape and therefore all the momentum anisotropy would stem from the anisotropic escape mechanism due to surface bias effects. The time evolution of energy density of the system is therefore governed only by the Bjorken scaling solution [32],

$$
\epsilon \propto \tau^{-4 / 3} \text {. }
$$

Using the above equation, the freeze-out time $\tau_{f}(x, y)$ can be obtained as a function of the initial energy density,

$$
\tau_{f}(x, y)=\tau_{i}\left[\frac{\epsilon_{i}(x, y)}{\epsilon_{f}}\right]^{3 / 4},
$$

where $\tau_{i}$ is the initialization time, $\epsilon_{f}$ is the freeze-out energy density, and $\epsilon_{i}(x, y)$ the initial energy density distribution obtained using the optical Glauber model of nuclear collisions.

The emitted hadron spectra can be obtained using the Cooper-Frye prescription for particle production [33],

$$
\frac{d N}{d^{2} p_{T} d y}=\frac{g}{(2 \pi)^{3}} \int p_{\mu} d \Sigma^{\mu} f(x, p),
$$

where $g$ is the degeneracy factor, $d \Sigma_{\mu}$ is the oriented freezeout hyper-surface and $f(x, p)$ is the phase-space distribution function of the particles at freeze-out. In the present case we use a classical Maxwell-Boltzmann distribution function for simplicity, $f_{0}=\exp \left(-u_{\mu} p^{\mu} / T\right)$. The above prescription should be modified to account for the re-absorption of the hadrons in the medium due to surface bias effects. We propose the following modification:

$$
\frac{d N^{T}}{d^{2} p_{T} d y}=\frac{g}{(2 \pi)^{3}} \int p_{\mu} d \Sigma^{\mu} \Theta(x, p) f(x, p),
$$

where $\Theta(x, p)$ is the transmittance coefficient which can, in general, depend on the position and momentum of the produced hadrons.

The components of particle four-momenta, $p^{\mu}$, are given by

$$
\begin{aligned}
p^{\tau} & =m_{T} \cosh \left(y-\eta_{s}\right), \\
p^{x} & =p_{T} \cos \phi, \quad p^{y}=p_{T} \sin \phi, \\
p^{\eta_{s}} & =m_{T} \sinh \left(y-\eta_{s}\right) / \tau,
\end{aligned}
$$

where $m_{T}^{2}=p_{T}^{2}+m^{2}, \quad p_{T}$ is the transverse momentum, $y$ is the particle rapidity, and $\phi$ is the azimuthal angle in the momentum space. The freeze-out hypersurface can be written as $d \Sigma_{\mu}=\left(m_{T} \cosh \eta_{s},-\partial \tau_{f} / \partial x\right.$, $\left.-\partial \tau_{f} / \partial y, m_{T} \sinh \eta_{s}\right) \tau_{f} d \eta_{s} d x d y$, where $\tau_{f}(x, y)$ is the freeze-out time. Moreover, we note that in the absence of transverse expansion, $u_{\mu} p^{\mu}=m_{T} \cosh \left(y-\eta_{s}\right)$ and

$$
\begin{aligned}
p^{\mu} d \Sigma_{\mu}= & {\left[m_{T} \cosh \left(y-\eta_{s}\right)-\left(p^{x} \frac{\partial \tau_{f}}{\partial x}+p^{y} \frac{\partial \tau_{f}}{\partial y}\right)\right] } \\
& \times \tau_{f} d \eta_{s} d x d y .
\end{aligned}
$$

In the above equation, the derivatives of $\tau_{f}(x, y)$ indicate the curvature of the constant temperature freeze-out hyper-surface.

For longitudinal boost-invariant flow where the transverse expansion is ignored, the spectra of emitted particles are

$$
\begin{aligned}
\frac{d N}{d^{2} p_{T} d y}= & \frac{g}{4 \pi^{3}}\left[m_{T} K_{1} \int \tau_{f} d x d y\right. \\
& \left.-K_{0} \int\left(p^{x} \frac{\partial \tau_{f}}{\partial x}+p^{y} \frac{\partial \tau_{f}}{\partial y}\right) \tau_{f} d x d y\right],
\end{aligned}
$$

where $K_{n} \equiv K_{n}\left(z_{m}\right)$ are the modified Bessel functions of the second kind of order $n$ with argument $z_{m} \equiv m_{T} / T_{f}$ and $T_{f}$ is the freeze-out temperature corresponding to the freeze-out 
energy density $\epsilon_{f}$. Note that for the spectra of emitted hadrons given in Eq. (7), the anisotropic flow defined as

$$
v_{n}\left(p_{T}\right) \equiv \frac{\int_{-\pi}^{\pi} d \phi \cos (n \phi) \frac{d N}{d y p_{T} d p_{T} d \phi}}{\int_{-\pi}^{\pi} d \phi \frac{d N}{d y p_{T} d p_{T} d \phi}}
$$

vanishes.

The hadrons produced using Eq. (7) are emitted isotropically in $\phi$. However, it is important to note that the hadrons that have to traverse through the medium may be reabsorbed. Therefore, one should take into account the probability of absorption of the hadrons in the medium. The probability of escape of the produced hadrons is given by the Beer-Lambert law for transmittance, $P_{\mathrm{esc}}=\exp \left(-\int \rho \sigma d l\right)$, where $\rho$ is the space-time dependent density of the system and $\sigma$ is interaction cross section. One should keep in mind that the transmittance probability involves the entire future density evolution of the system and is not captured by the Cooper-Frye freeze-out prescription.

In the present work, we use a modified version of the BeerLambert law for transmittance. We consider the energy-density dependent attenuation of the hadrons traveling through the medium in the transverse plane. The transmittance probability is given by

$$
\Theta\left(x, y, p_{T}, \phi\right)=\exp \left[-\int_{0}^{\infty} \frac{\sigma\left(p_{T}\right)}{T} \epsilon\left(x^{\prime}, y^{\prime} ; \tau^{\prime}\right) d l\right],
$$

where $x^{\prime}=x+l \cos \phi, y^{\prime}=y+l \sin \phi$, and $\tau^{\prime}$ is the time at which the hadrons reach the point $\left(x^{\prime}, y^{\prime}\right)$ in the transverse plane. In the above equation, we consider a constant cross section for the hadrons interacting with the medium,

$$
\sigma=\frac{T}{\epsilon_{f} \Lambda}
$$

where $\Lambda$ is the length scale associated with the absorption of the hadrons inside the medium. The above form of $\sigma$ is motivated by transport calculations where one assumes constant cross sections for the partonic and hadronic interactions.

Next we consider the evolution of the medium while the hadrons are traversing through it. The time at which the hadrons are produced is $\tau_{f}$ and the time at which they traverse a length $l$ to reach the point $\left(x^{\prime}, y^{\prime}\right)$ is

$$
\tau^{\prime}=\tau_{f}+\frac{l}{v_{T}}, \text { where } v_{T}=\frac{p_{T}}{m_{T}} .
$$

Using the Bjorken scaling, Eq. (1), we can obtain the energy density at time $\tau^{\prime}$ to be

$$
\epsilon\left(x^{\prime}, y^{\prime} ; \tau^{\prime}\right)=\epsilon\left(x^{\prime}, y^{\prime} ; \tau_{f}\right)\left(1+\frac{l m_{T}}{\tau_{f} p_{T}}\right)^{-4 / 3} .
$$

Using the Bjorken scaling relation again, one can obtain $\epsilon\left(x^{\prime}, y^{\prime} ; \tau_{f}\right)$ in terms of the initial energy density distribution,

$$
\epsilon\left(x^{\prime}, y^{\prime} ; \tau_{f}\right)=\epsilon_{i}\left(x^{\prime}, y^{\prime}\right)\left[\frac{\tau_{i}}{\tau_{f}(x, y)}\right]^{4 / 3}=\frac{\epsilon_{i}\left(x^{\prime}, y^{\prime}\right)}{\epsilon_{i}(x, y)} \epsilon_{f},
$$

where we have used Eq. (2) to write the last equality.
Using Eqs. (10), (12), and (13), we see that Eq. (9) can be written as

$$
\begin{aligned}
\Theta\left(x, y, p_{T}, \phi\right)= & \exp \left[-\frac{1}{\epsilon_{i}(x, y) \Lambda} \int_{0}^{\infty} d l \epsilon_{i}(x+l \cos \phi, y\right. \\
& \left.+l \sin \phi)\left(1+\frac{l m_{T}}{\tau_{f} p_{T}}\right)^{-4 / 3}\right] .
\end{aligned}
$$

This is the final form of the transmittance coefficient which can be used in Eq. (4) to calculate the transmitted spectra of hadrons.

The spectra of transmitted hadrons, obtained after CooperFrye freeze-out in Eq. (7) with the transmittance probability proposed in Eq. (4), can be written as

$$
\begin{aligned}
\frac{d N^{T}}{d^{2} p_{T} d y}= & \frac{g}{4 \pi^{3}}\left[m_{T} K_{1} \int \Theta\left(x, y, p_{T}, \phi\right) \tau_{f} d x d y\right. \\
& -K_{0} \int \Theta\left(x, y, p_{T}, \phi\right)\left(p^{x} \frac{\partial \tau_{f}}{\partial x}+p^{y} \frac{\partial \tau_{f}}{\partial y}\right) \\
& \left.\times \tau_{f} d x d y\right] .
\end{aligned}
$$

Note that the spectra of transmitted hadrons now has $\phi$ dependence via the transmittance coefficient $\Theta$ and can lead to sizable anisotropic flow,

$$
v_{n}\left(p_{T}\right) \equiv \frac{\int_{-\pi}^{\pi} d \phi \cos (n \phi) \frac{d N^{T}}{d y p_{T} d p_{T} d \phi}}{\int_{-\pi}^{\pi} d \phi \frac{d N^{T}}{d y p_{T} d p_{T} d \phi}} .
$$

In the present work, we focus only on the second harmonic of the anisotropic flow, $v_{2}\left(p_{T}\right)$, also known as the elliptic flow. This is the most dominant flow harmonic for noncentral collisions and depends predominantly on the initial geometrical shape of the medium.

Here we point out some limitations of our model. We neglect the energy deposited in the medium by the absorbed hadrons and therefore the change in energy density of the medium. Since the hadrons are soft probes, the heating of the medium due to deposited energy can be safely neglected compared to cooling due to Bjorken expansion and, therefore, is not important for the current study. Another assumption is that we do not account for the change in the momentum of the emitted hadrons; i.e., we assume that the hadrons are either absorbed or transmitted completely without energy loss. While it is difficult to account for the momentum loss of the hadrons, the uncertainty due to this on $v_{2}$ should not be large. We leave this study for future work.

\section{RESULTS AND DISCUSSIONS}

As a demonstration of our model, we apply it to calculate the elliptic flow of charged hadrons for $\mathrm{Au}+\mathrm{Au}$ collisions at $\sqrt{s_{N N}}=200 \mathrm{GeV}$ at RHIC and $\mathrm{Pb}+\mathrm{Pb}$ collisions at $\sqrt{s_{N N}}=$ $2.76 \mathrm{TeV}$ at LHC. In Fig. 1, we show the $p_{T}$ dependence of $v_{2}$, obtained from Eq. (16), for various centralities at RHIC. The symbols in Fig. 1 are experimental results obtained by the PHENIX and STAR Collaborations [34-36]. Theoretical 


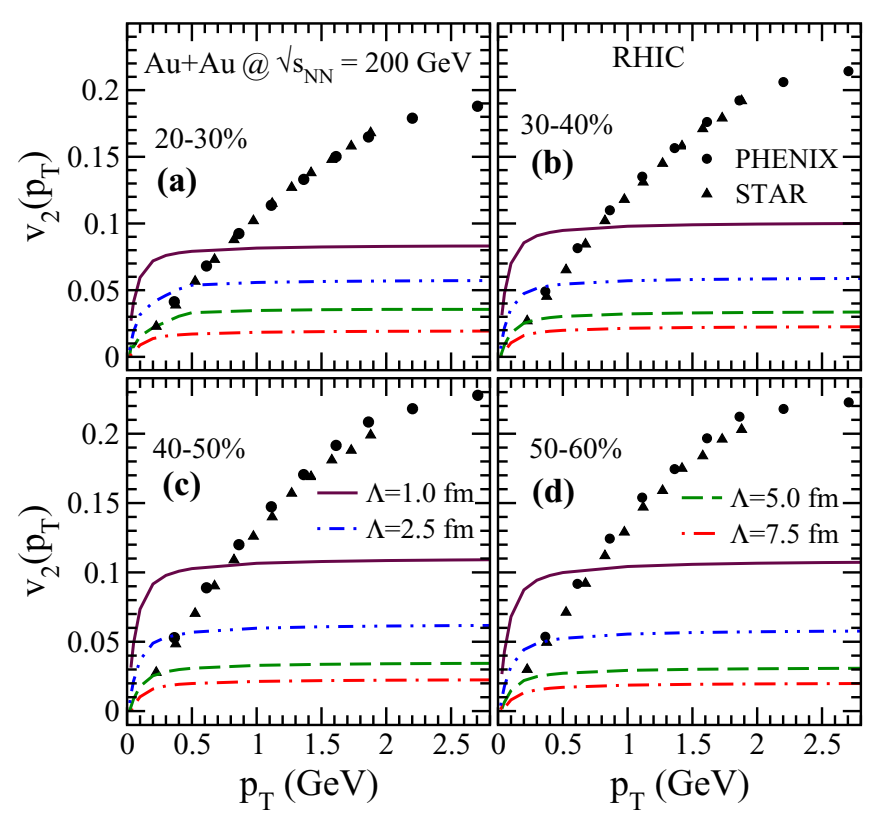

FIG. 1. Transverse momentum dependence of elliptic flow of charged hadrons for RHIC at various centralities. The symbols represent experimental results from PHENIX and STAR Collaborations. The theoretical curves are obtained by considering various values of attenuation length of hadrons in the medium. The error bars for the experimental data are contained within the symbol size.

curves are generated for different values: $\Lambda=1,2.5,5$, and $7.5 \mathrm{fm}$. For RHIC, the initial energy density at the center of the fireball is set to $\epsilon_{i}(0,0)=30 \mathrm{GeV} / \mathrm{fm}^{3}$ and the initial thermalization time is taken as $\tau_{i}=0.6 \mathrm{fm} / c$ [37]. We consider freeze-out at energy density corresponding to a temperature of $T_{f}=130 \mathrm{MeV}$. The results for $\mathrm{Pb}+\mathrm{Pb}$ collisions at LHC at $\sqrt{s_{N N}}=2.76 \mathrm{TeV}$ are depicted in Fig. 2 for similar values of $\Lambda$. The symbols in Fig. 2 represent experimental results obtained by the ATLAS Collaboration [38]. The other parameters for LHC are taken as $\epsilon_{i}(0,0)=85 \mathrm{GeV} / \mathrm{fm}^{3}$, and $\tau_{i}=0.4 \mathrm{fm} / c$ [39]. The freeze-out energy density is taken to be the same as that at RHIC.

In Figs. 1 and 2, we observe that for very small transverse momentum, $p_{T} \leqslant 0.1 \mathrm{GeV}$, the anisotropic escape mechanism does not generate any elliptic flow. This may be attributed to the fact that, for very low energy hadrons, the medium expands faster than their propagation speed and the hadrons do not experience the shape anisotropy of the fireball. However, for hadrons above a certain transverse momentum, they are attenuated by a constant factor depending on the interaction cross section and hence the absorption length scale, $\Lambda$, in the medium. For small values of $\Lambda$, the attenuation is larger and therefore larger elliptic flow is generated. However, we observe that, even for a very small attenuation length, $\Lambda=1$ fm (solid brown curves), the model is unable to generate the magnitude of experimentally observed elliptic flow. This shows that anisotropic escape is not sufficient to explain the experimental data and one needs hydrodynamic buildup of collective flow to get the correct magnitude of the observed elliptic flow. However, we see that the elliptic flow generated from

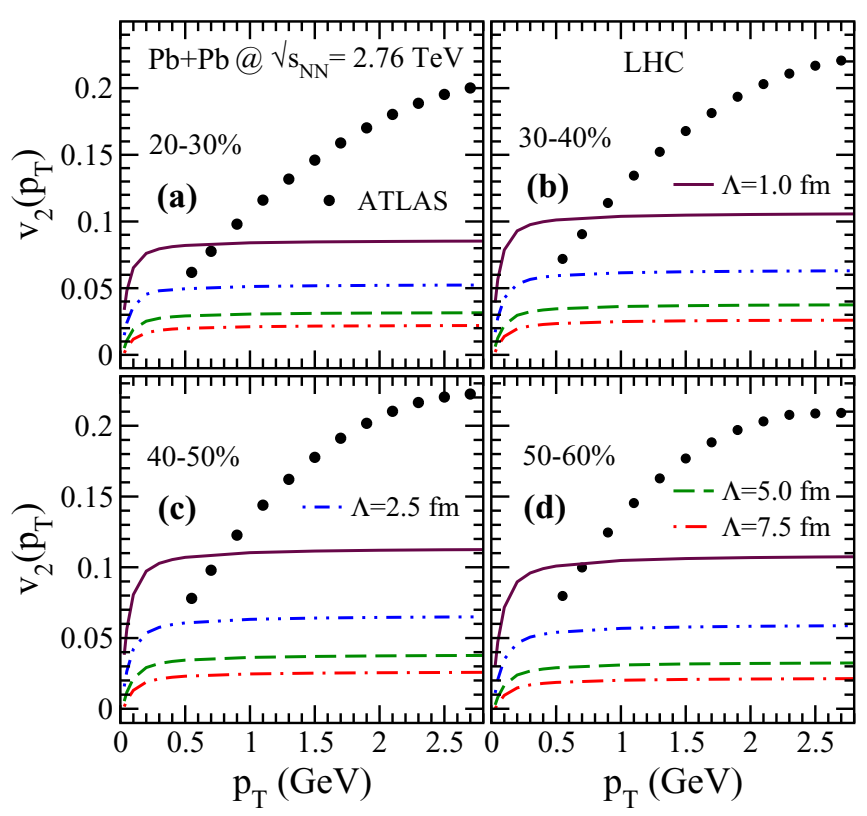

FIG. 2. Transverse momentum dependence of elliptic flow of charged hadrons for LHC at various centralities. The symbols represent experimental results from the ATLAS Collaboration. The theoretical curves are obtained by considering various values of attenuation length of hadrons in the medium. The error bars for the experimental data are contained within the symbol size.

anisotropic escape is non-negligible and should be corrected for in the Cooper-Frye prescription for freeze-out.

It is interesting to note that the $p_{T}$ dependence of $v_{2}$ in Figs. 1 and 2 is similar to that obtained in Ref. [28], where it was shown that, within a Boltzmann kinetic approach, a single collision per particle is enough to generate sizable elliptic flow. However, we find that, within the present model, the escape mechanism alone is not enough to generate the observed elliptic flow in non-central heavy-ion collisions. This is contrary to the findings of Ref. [10], where the authors claim that the majority of anisotropic flow is generated from an escape mechanism within a Boltzmann transport approach. This may be attributed to the fact that while we consider an equilibrated system, the parton-parton interactions in the transport calculations may not be enough for the system to attain equilibrium. However, when the parton-parton interaction cross section is increased, the authors of Ref. [10] find that the hydrodynamic-type contribution to the anisotropic flow starts to dominate over the contribution due to the escape mechanism, which is in agreement with our findings. Therefore, we provide an extremely simple model to account for the escape mechanism which can easily be implemented in the Cooper-Frye freeze-out prescription in a realistic hydrodynamic calculation.

\section{SUMMARY AND CONCLUSION}

In this paper, we studied the effect of an anisotropic escape mechanism due to source shape anisotropy on elliptic flow in relativistic heavy-ion collisions. We have used an optical Glauber model to generate initial conditions and ignored hydrodynamic expansion in the transverse direction to separate 
out non-flow contributions from surface bias effects. In order to calculate the probability of transmittance of produced hadrons through the medium, we have employed the Beer-Lambert law. We found that this method, to account for loss of hadrons inside the medium, leads to a final momentum anisotropy of the observed hadrons even though the transverse fluid velocity vanishes. As a demonstration, we calculated the transverse momentum dependence of elliptic flow, generated from an anisotropic escape mechanism due to surface bias effects, for various centralities in $\sqrt{s_{N N}}=200 \mathrm{GeV} \mathrm{Au}+\mathrm{Au}$ collisions at RHIC and $\sqrt{s_{N N}}=2.76 \mathrm{TeV} \mathrm{Pb}+\mathrm{Pb}$ collisions at LHC. We found that the surface bias effects have a sizable contribution to the total elliptic flow observed in these collisions. However, the elliptic flow generated from the escape mechanism alone is not sufficient to explain the experimental data. This indicates that, while hydrodynamic buildup of fluid velocity is necessary to get the correct magnitude of elliptic flow, the viscosity of the QCD matter extracted from hydrodynamic simulations may be underestimated.

At this juncture, it is important to point out that in the presence of transverse fluid velocity, the number of hadrons entering the medium is relatively less as the momentum of the hadrons is biased towards the outward direction. However, this effect becomes more important when one has fluctuations in the initial condition leading to irregularities in the freeze-out hyper-surface. This leads to negative Cooper-Frye contributions [40] and hence one should correctly account for the suppression of these hadrons in a realistic hydrodynamic calculation after freeze-out. While this is left for future work, we note that the escape mechanism might play a dominant role in small systems where the formation of a hydrodynamic medium is questionable. Therefore, it will be interesting to explore the applicability of the present model to generate flow in small systems.

\section{ACKNOWLEDGMENTS}

The authors would like to thank Volker Koch for useful discussions during the initial stages of this work. We thank Victor Roy and Debojit Sarkar for helpful comments. The authors gratefully acknowledge support from GSI Darmstadt where this project was initiated. A.J. is supported in part by the DST-INSPIRE faculty award under Grant No. DST/INSPIRE/04/2017/000038.
[1] P. Braun-Munzinger, V. Koch, T. Schäfer, and J. Stachel, Properties of hot and dense matter from relativistic heavy ion collisions, Phys. Rep. 621, 76 (2016).

[2] U. Heinz and R. Snellings, Collective flow and viscosity in relativistic heavy-ion collisions, Annu. Rev. Nucl. Part. Sci. 63, 123 (2013)

[3] C. Gale, S. Jeon, and B. Schenke, Hydrodynamic modeling of heavy-ion collisions, Int. J. Mod. Phys. A 28, 1340011 (2013).

[4] A. Jaiswal and V. Roy, Relativistic hydrodynamics in heavy-ion collisions: General aspects and recent developments, Adv. High Energy Phys. 2016, 9623034 (2016).

[5] W. Florkowski, M. P. Heller, and M. Spalinski, New theories of relativistic hydrodynamics in the LHC era, Rep. Prog. Phys. 81, 046001 (2018).

[6] P. K. Kovtun, D. T. Son, and A. O. Starinets, Viscosity in Strongly Interacting Quantum Field Theories from Black Hole Physics, Phys. Rev. Lett. 94, 111601 (2005).

[7] Y. Zhou, X. Zhu, P. Li, and H. Song, Investigation of possible hadronic flow in $\sqrt{s_{N N}}=5.02 \mathrm{TeV} p-\mathrm{Pb}$ collisions, Phys. Rev. C 91, 064908 (2015).

[8] J. D. Orjuela Koop, A. Adare, D. McGlinchey, and J. L. Nagle, Azimuthal anisotropy relative to the participant plane from a multiphase transport model in central $p+\mathrm{Au}, d+\mathrm{Au}$, and ${ }^{3} \mathrm{He}$ + Au collisions at $\sqrt{s_{N N}}=200 \mathrm{GeV}$, Phys. Rev. C 92, 054903 (2015).

[9] P. Bozek, A. Bzdak, and G. L. Ma, Rapidity dependence of elliptic and triangular flow in proton-nucleus collisions from collective dynamics, Phys. Lett. B 748, 301 (2015).

[10] L. He, T. Edmonds, Z. W. Lin, F. Liu, D. Molnar, and F. Wang, Anisotropic parton escape is the dominant source of azimuthal anisotropy in transport models, Phys. Lett. B 753, 506 (2016).

[11] P. Romatschke, Collective flow without hydrodynamics: Simulation results for relativistic ion collisions, Eur. Phys. J. C 75, 429 (2015).
[12] P. Romatschke, How to fake hydrodynamic signals, Nucl. Phys. A 956, 222 (2016).

[13] A. Adare et al. (PHENIX Collaboration), Measurement of LongRange Angular Correlation and Quadrupole Anisotropy of Pions and (Anti)protons in Central $d+$ Au Collisions at $\sqrt{s_{N N}}=200$ GeV, Phys. Rev. Lett. 114, 192301 (2015).

[14] B. Abelev et al. (ALICE Collaboration), Long-range angular correlations on the near and away side in $p-\mathrm{Pb}$ collisions at $\sqrt{s_{N N}}=5.02 \mathrm{TeV}$, Phys. Lett. B 719, 29 (2013).

[15] B. B. Abelev et al. (ALICE Collaboration), Long-range angular correlations of $\pi, K$ and $p$ in $p$-Pb collisions at $\sqrt{s_{\mathrm{NN}}}=5.02$ TeV, Phys. Lett. B 726, 164 (2013).

[16] B. B. Abelev et al. (ALICE Collaboration), Multiplicity dependence of pion, kaon, proton and lambda production in $\mathrm{p}-\mathrm{Pb}$ collisions at $\sqrt{s_{N N}}=5.02 \mathrm{TeV}$, Phys. Lett. B 728, 25 (2014).

[17] B. B. Abelev et al. (ALICE Collaboration), Multiparticle azimuthal correlations in $p-\mathrm{Pb}$ and $\mathrm{Pb}-\mathrm{Pb}$ collisions at the CERN Large Hadron Collider, Phys. Rev. C 90, 054901 (2014).

[18] D. Sarkar, S. Choudhury, and S. Chattopadhyay, Twoparticle correlations with identified triggers in $p$ - $\mathrm{Pb}$ collisions at $\sqrt{s_{N N}}=5.02 \mathrm{TeV}$ using a multiphase transport model, Phys. Rev. C 94, 044909 (2016).

[19] D. Sarkar, S. Choudhury, and S. Chattopadhyay, Effect of radial flow on two particle correlations with identified triggers at intermediate $\mathrm{p}_{T}$ in $p$ - $\mathrm{Pb}$ collisions at $\sqrt{s_{N N}}=5.02 \mathrm{TeV}$, Phys. Lett. B 760, 763 (2016).

[20] D. Sarkar and S. Chattopadhyay, Ridge from jet-medium interaction in $p$-Pb collisions at $\sqrt{s_{N N}}=5.02 \mathrm{TeV}$, Phys. Rev. C 95, 044906 (2017).

[21] P. Ghosh, S. Muhuri, J. K. Nayak, and R. Varma, Indication of transverse radial flow in high-multiplicity proton-proton collisions at the Large Hadron Collider, J. Phys. G 41, 035106 (2014). 
[22] T. Kalaydzhyan and E. Shuryak, Collective flow in highmultiplicity proton-proton collisions, Phys. Rev. C 91, 054913 (2015).

[23] I. Bautista, A. F. Téllez, and P. Ghosh, Indication of change of phase in high-multiplicity proton-proton events at LHC in string percolation model, Phys. Rev. D 92, 071504 (2015).

[24] L. J. Gutay, A. S. Hirsch, C. Pajares, R. P. Scharenberg, and B. K. Srivastava, De-confinement in small systems: Clustering of color sources in high multiplicity $\bar{p}$ p collisions at $\sqrt{s}=1.8$ TeV, Int. J. Mod. Phys. E 24, 1550101 (2015).

[25] J. D. Orjuela Koop, R. Belmont, P. Yin, and J. L. Nagle, Exploring the beam-energy dependence of flow-like signatures in small system $d+$ Au collisions, Phys. Rev. C 93, 044910 (2016).

[26] D. Sarkar, S. Choudhury, and S. Chattopadhyay, Investigating the role of partonic and hadronic dynamics in mass splitting of elliptic anisotropy in $p$ - $\mathrm{Pb}$ collisions at $\sqrt{s_{N N}}=5.02 \mathrm{TeV}$, Phys. Rev. C 94, 044919 (2016).

[27] B. Tomasik and U. A. Wiedemann, Freezeout mechanism and phase space density in ultrarelativistic heavy ion collisions, Phys. Rev. C 68, 034905 (2003).

[28] N. Borghini and C. Gombeaud, Anisotropic flow far from equilibrium, Eur. Phys. J. C 71, 1612 (2011).

[29] S. K. Das and J. E. Alam, Elliptic flow of heavy flavors, arXiv:1008.2643.

[30] S. K. Das and J. E. Alam, Heavy flavour suppression and flow, arXiv:1101.3385.

[31] J. Liao and V. Koch, Exposing the Non-Collectivity in Elliptic Flow, Phys. Rev. Lett. 103, 042302 (2009).

[32] J. D. Bjorken, Highly relativistic nucleus-nucleus collisions: The central rapidity region, Phys. Rev. D 27, 140 (1983).
[33] F. Cooper and G. Frye, Comment on the single particle distribution in the hydrodynamic and statistical thermodynamic models of multiparticle production, Phys. Rev. D 10, 186 (1974).

[34] A. Adare et al. (PHENIX Collaboration), Measurements of Higher-Order Flow Harmonics in Au+Au Collisions at $\sqrt{s_{N N}}=$ $200 \mathrm{GeV}$, Phys. Rev. Lett. 107, 252301 (2011).

[35] J. Adams et al. (STAR Collaboration), Azimuthal anisotropy in $\mathrm{Au}+\mathrm{Au}$ collisions at $\sqrt{s_{N N}}=200 \mathrm{GeV}$, Phys. Rev. C 72, 014904 (2005).

[36] L. Adamczyk et al. (STAR Collaboration), Third harmonic flow of charged particles in $\mathrm{Au}+\mathrm{Au}$ collisions at $\sqrt{s_{N N}}=200 \mathrm{GeV}$, Phys. Rev. C 88, 014904 (2013).

[37] S. S. Adler et al. (PHENIX Collaboration), Systematic studies of the centrality and $\sqrt{s_{N N}}$ dependence of the $d E_{T} / d \eta$ and $d N_{\mathrm{ch}} / d \eta$ in heavy ion collisions at mid-rapidity, Phys. Rev. C 71, 034908 (2005); Systematic studies of the centrality and $\sqrt{s_{N N}}$ dependence of the $d E_{T} / d \eta$ and $d N_{\mathrm{ch}} / d \eta$ in heavy ion collisions at mid-rapidity [Phys. Rev. C 71, 034908 (2005)] 71, 049901(E) (2005).

[38] G. Aad et al. (ATLAS Collaboration), Measurement of the azimuthal anisotropy for charged particle production in $\sqrt{s_{N N}}=$ 2.76 TeV, Phys. Rev. C 86, 014907 (2012).

[39] B. Abelev et al. (ALICE Collaboration), Centrality dependence of $\pi, K$, and $p$ production in $\mathrm{Pb}-\mathrm{Pb}$ collisions at $\sqrt{s_{N N}}=2.76$ TeV, Phys. Rev. C 88, 044910 (2013).

[40] D. Oliinychenko, P. Huovinen, and H. Petersen, Systematic investigation of negative Cooper-Frye contributions in heavy ion collisions using coarse-grained molecular dynamics, Phys. Rev. C 91, 024906 (2015). 